\title{
Anticancer Therapy Based on Suppression of Pathways Recruited to Cope with Metabolic Stress
}

\author{
Fernández $\mathbf{A}^{1,2 *}$ \\ ${ }^{1}$ Argentine Institute of Mathematics (I.A.M.), National Research Council (CONICET), Buenos Aires 1083, Argentina \\ ${ }^{2}$ Collegium Basilea, Institute for Advanced Study, Hochstrasse 51, Basel 4053, Switzerland
}

It is widely felt that the neglect of biochemical aspects of cancer may have significantly hampered progress towards an effective cure of the disease. In the days of Otto Warburg, the biochemical focus was present and in fact dominant in molecular approaches to anticancer therapy [1]. However, this view began to fell out of favor as the genetic basis of the disease was established with the discovery of the first oncogene in Robert Weinberg's lab at MIT. Strikingly, a landmark contemporary book like Weinberg's "The Biology of Cancer” [2] hardly contains any reference to the biochemical etiology and biochemical mechanisms of the disease.

Here we advocate for a revival of the biochemical approach since much has happened lately in cancer research that could revitalize the views originally spearheaded by Warburg. This is especially timely given the phenomenal progress in regards to the delineation of the signaling pathways that control and regulate the enzymatic of cancer metabolism.

Since the days of Otto Warburg, it has been noted that cancer cells have much higher energetic demands than normal cells, and this makes them vulnerable to metabolic stress [3]. The sensitivity of cancer cells to sustained decreases in the AMP/ATP ratio is now widely established, and recent therapeutic strategies are attempting to harness this fact [3]. In this regard, the type-2 diabetes drug metformin is being carefully evaluated as an anticancer agent due to its inhibitory impact on the mitochondrial respiratory chain, suppression of hepatic glucose production and decrease of the insulin-induced suppression of fatty acid oxidation. It is now well established that metformin inhibits the electron transport chain complex 1 , thus becoming a major exogenous disruptor of energy homeostasis in the cell.

Yet, the efficacy of metformin as an anticancer agent per se is heavily compromised by its agonistic action on the kinase AMPK, a major regulator of energy homeostasis. The functionally competent AMPK complex is allosterically activated by AMP and inhibited by ATP, thus becoming a sensor of energetic imbalance. When activated, AMPK inhibits anabolic processes, such as fatty acid synthesis and protein synthesis, while promoting catabolic pathways for fatty acid oxidation and glucose transport through vesicle $\rightarrow$ plasma membrane translocation of GLUT4. Thus, increase in AMPK activation levels helps the cell cope with metabolic stress. In this context, AMPK becomes clearly an oncogene, in sharp contrast with the tumor suppressor role often attributed to it [3]. On the other hand, metformin is an AMPK agonist simply because it alters the AMP/ATP ratio, but has no direct effect on AMPK. This agonistic role severely impairs the efficacy of metformin as an anticancer agent since it turns on the metabolic switch that enables cancer cells to cope with the metabolic stress induced by metformin.

Ergo, to position metformin, or simply intense physical exercise, as anticancer agents acting by imposing metabolic stress on the cancercell we need to edit out the agonistic effect exerted by these agents upon AMPK. In other words, we need to combine metformin or intense physical exercise with an AMPK inhibitor that would act as a therapeutic editor by suppressing the stress-coping mechanism triggered by the allosteric activation of AMPK. This combination strategy is likely to work, since it is well established that the loss of energy homeostasis leads to apoptosis in cells lacking AMPK [3].

There are several chemical leads that may be potentially redesigned to turn them into selective AMPK inhibitors. The most interesting lead is TAE-684, a powerful AMPK inhibitor whose value as therapeutic editor is severely compromised due to its undesired cross reactivity against the insulin receptor (InsR) kinase [4]. It would be necessary to exploit the selectivity filters provided by epistructural analysis [5] to dial out the anti-InsR activity of TAE-684, while retaining or possibly enhancing its inhibitory impact against AMPK through wrappingbased redesign.

This research was made possible through a grant from AF Innovation, a pharmaceutical consultancy.

\section{References}

1. Warburg $\mathrm{O}$ (1956) On the Origin of Cancer Cells. Science 123: 309-314.

2. Weinberg RA (2013) The Biology of Cancer. New York.

3. Liang J, Mills GB (2013) AMPK: A Contextual Oncogene or Tumor Suppressor? Cancer Res 73: 2929-2935.

4. Karaman MW, Herrgard S, Treiber DK, Gallant P, Atteridge CE, et al. (2008) A quantitative analysis of kinase inhibitor selectivity. Nature Biotech 26:127-132

5. Fernandez Stigliano A (2015) Biomolecular Interfaces: Interactions, Functions and Drug Design. Springer, Berlin.
*Corresponding author: Fernández A, Argentine Institute of Mathematics (I.A.M.), National Research Council (CONICET), Buenos Aires 1083, Argentina, Tel: +34 981-780505; Fax: +34-981-780511; E-mail: ariel@afinnovation.com

Received January 20, 2016; Accepted January 25, 2016; Published January 27, 2016

Citation: Fernández A (2016) Anticancer Therapy Based on Suppression of Pathways Recruited to Cope with Metabolic Stress. Metabolomics 6: e144. doi:10.4172/2153-0769.1000e144

Copyright: ( 2016 Fernández A. This is an open-access article distributed unde the terms of the Creative Commons Attribution License, which permits unrestricted use, distribution, and reproduction in any medium, provided the original author and source are credited. 